\title{
Research Article \\ OFDM Synchronization Errors and Effects of Phase Noise on WLAN Transceivers
}

\author{
Mourad Melliti, Salem Hasnaoui, and Ridha Bouallegue \\ Système de Communications Sys'Com Laboratoire, Ecole Nationale d'Ingénieurs de Tunis, 1002 Tunis, Tunisia
}

Correspondence should be addressed to Mourad Melliti, mourad.melliti@enit.rnu.tn

Received 29 January 2008; Revised 22 May 2008; Accepted 28 December 2008

Recommended by Jie Li

\begin{abstract}
This work aims at frequency synchronization in OFDM IEEE 802.11g Transceivers. The degradation of performance results from the detrimental effects introduced by frequency offset and phase noise. First, we present a robust method to detect and synchronize the OFDM signal with severe noise and interference corruption based on calculating the accumulated phase difference for each subcarrier. Then, we present a simple architecture to implement the proposed methods. These methods are simulated and analyzed by computer. Finally, we propose many algorithms to correct the common phase error (CPE) of the phase noise and track well its variation.
\end{abstract}

Copyright (C 2008 Mourad Melliti et al. This is an open access article distributed under the Creative Commons Attribution License, which permits unrestricted use, distribution, and reproduction in any medium, provided the original work is properly cited.

\section{INTRODUCTION}

Orthogonal frequency division multiplexing (OFDM) has been gaining popularity in a variety of digital communication systems, such as wireless LAN 802.11a and 802.11g. The imperfect synchronization between the tuner's local oscillation in transmitter and in receiver brings not only frequency offset but also phase noise. Many algorithms have been put forward for estimating and correcting common phase error (CPE). This paper proposes a novel adaptive algorithm for correcting CPE. The simulation results show that the algorithm is of good performance in CPE estimation.

After a brief introduction to OFDM systems in Section 2, this paper will be concerned in defining the general problem of improper frequency synchronization. In Sections 3 and 4 , we will explain why frequency offsets have detrimental effects on the received signal's spectrum. We will quantify the offset with respect to the carrier frequency spacing and will consider the two cases where the offset is either an integer multiple of the carrier spacing or not. Depending on this condition, we will see how the orthogonality among the carriers may be destroyed. We will then turn to study the effects of phase noise. We will characterize phase noise by describing the 2 types of phase error that it induces, namely, common phase error (CPE) and intercarrier interference
(ICI). The description will fully explain the modification induced by each type on the received signal's spectrum. In Section 7, we will briefly discuss the common approaches to remove the effects of $\mathrm{CPE}$.

\section{OFDM SYSTEMS}

The main reasons OFDM was adopted in the wireless local area network (WLAN) standards IEEE 802.11a [1] and g [2] are its high spectral efficiency and ability to deal with frequency-selective fading and narrowband interference. However, the main disadvantage that might result is the induced intersymbol interference (ISI). OFDM addresses this problem within the more general framework of multicarrier modulation. The bit-stream is divided into substreams that are each sent with a lower data rate on an individual subchannel.

As in all digital communication systems, OFDM requires proper synchronization between the transmitter and the receiver. Both time and frequency synchronization influence the performance of OFDM systems. The subdivision of the initial symbol into $\mathrm{N}$ parallel symbols results in a longer symbol duration. This will cause the system to be less sensitive to timing offsets. However, frequency offsets may heavily degrade the system's performance [1]. 


\section{IMPLEMENTATION OF QAM TRANSCEIVER}

By implementing a QAM-based transceiver, the OFDM signal is formed by first converting the frequency samples into time samples through the use of the inverse Fourier transform represented before by the IFFT [3].

The output time samples take the following form:

$$
S n=\frac{1}{\sqrt{N}} \sum_{k=0}^{N-1} S_{k} e^{j 2 \pi n k / N}, \quad n=0,1, \ldots, N-1 .
$$

These generated parallel time samples are then converted to a serial stream through a parallel-to-serial converter. The last step required before transmitting the stream over the channel is to add a cyclic prefix code. Indeed, one of the major properties of the continuous Fourier transform is the duality between convolution in time domain and multiplication in frequency domain. This property does not completely extend to the discrete case. For it to be correctly applied, one should carry circular convolution instead of the more common linear convolution in the time domain. To this end, we assume that the discrete channel impulse response has length $k$. Adding the cyclic prefix corresponds to appending the last $\mathrm{k}$ symbols of each block $\left[s_{0}, s_{1} \cdots s_{N}\right]$ to the beginning of the same block. We get the following block: $\left[s_{N-k}, s_{N-k-1} \cdots s_{0}, s_{1} \cdots s_{N}\right]$. This operation preserves the desired property of multiplication in the frequency domain. The resulting sequence is passed through a digital-to-analog converter and then up converted to a higher carrier frequency. The signal is then sent through the channel.

On the receiver end, noise introduced by the channel will be superimposed on the received signal. The signal is first down converted to base-band and then passed through a lowpass filter to remove the effects of high-frequency terms. The time samples are retrieved by passing the resulting signal through an analog-to-digital converter. The cyclic prefix is first removed from each stream and then the stream is converted to a parallel stream through a serial-to-parallel converter. The DFT is then performed to recover the original coefficients. These are then converted to a serial stream to form the symbol that was originally sent.

As can be clearly seen from Figure 1, the required overall bandwidth of an OFDM system is equal to $\mathrm{NB} / 2$. In this sense, OFDM systems make better use of the available system bandwidth.

\section{FREQUENCY SYNCHRONIZATION}

As in all digital communication modems, OFDM requires proper synchronization between the transmitter and the receiver. Both time and frequency synchronizations influence the performance of OFDM systems. The subdivision of the initial symbol into $N$ parallel symbols results in a longer symbol duration. This will cause the system to be less sensitive to timing offsets. However, frequency offsets may heavily degrade the system's performance. To see this, we first derive the spectrum of an OFDM symbol from its time domain representation. The time domain version of the

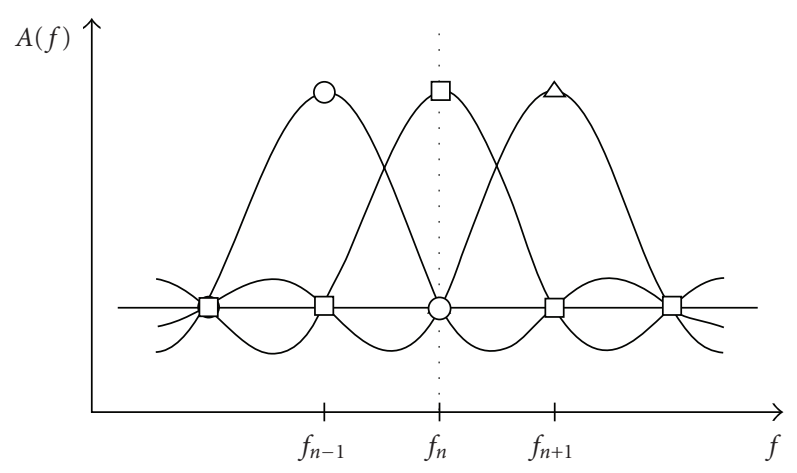

(a)

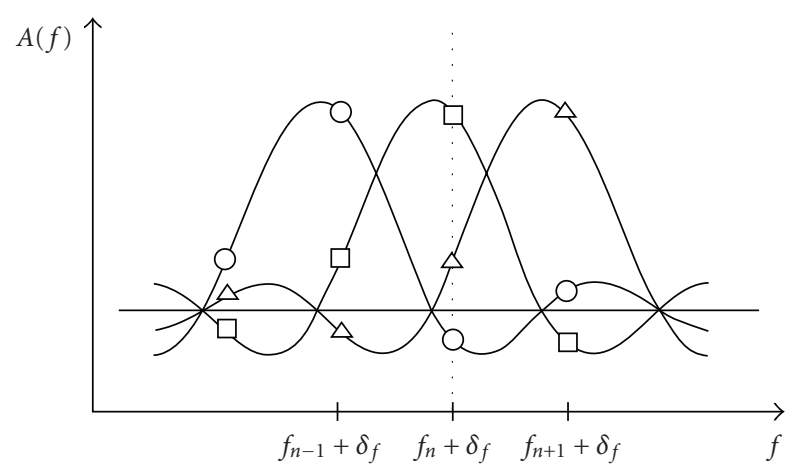

(b)

FIGURE 1: Effects of frequency shift on OFDM spectrum.

symbol is the one transmitted over the channel. We represent the transmitted OFDM symbol as follows:

$$
S(t)=\sum_{k=0}^{N-1} S_{k} e^{j 2 \pi\left(f_{0}+k \Delta_{f}\right) t} \operatorname{rect}\left(\frac{t}{N T_{s}}\right),
$$

$S_{k}$ denotes the complex amplitude of the $k$ th carrier, $\Delta_{f}$ is the carrier spacing, and $f_{0}$ is the frequency of the 0 th carrier. The multiplication by the window function is due to the fact that each OFDM symbol is time limited to a duration $T_{s}$.

We then compute the Fourier transform of the aforementioned time signal to get

$$
S(f)=\sum_{k=0}^{N-1} S_{k} \sin c\left(N T_{s} f\right) * \delta\left(f-\left(f_{0}+k \Delta_{f}\right)\right) .
$$

Now, we note that the total bandwidth is equal to $N \Delta_{f}$ and so the symbol time $T_{s}$ is equal to $1 / N \Delta_{f}$. Replacing $N T_{s}$ by $1 / \Delta_{f}$, we get

$$
S(f)=\sum_{k=0}^{N-1} S_{k} \sin c\left(\frac{f-\left(f_{0}+k \Delta_{f}\right)}{\Delta_{f}}\right) .
$$

It might happen that on the demodulator's side, the carriers get shifted by an amount $\delta_{f}$. The spacing between the carriers is still equal to $\Delta_{f}$. This can be seen from Figure 2 .

In this case, we have 3 subcarriers and the symbols on the 3 different curves correspond to the different contributions of each of the subcarriers to the sum of the sampling points. 


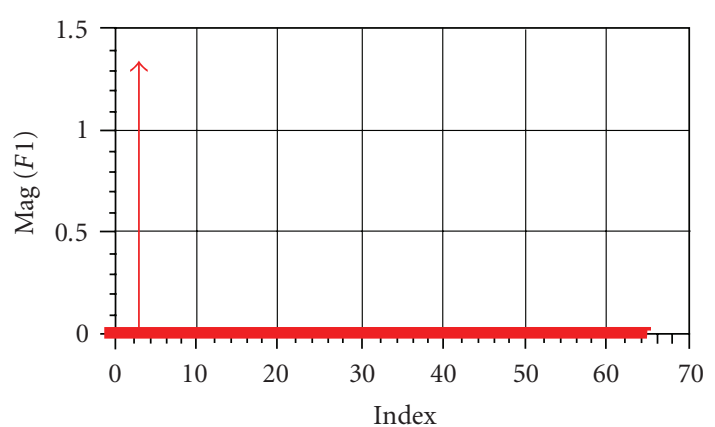

Symbol to be transmitted (magnitude spectrum)

(a)

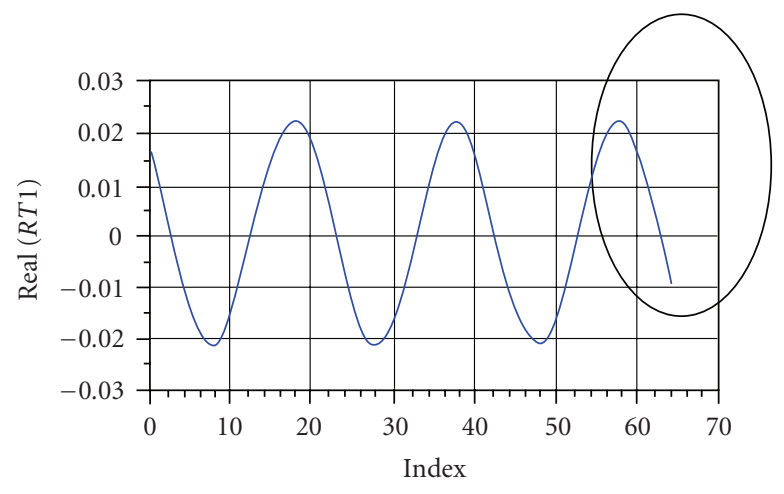

Demodulated I-channel signal

(c)

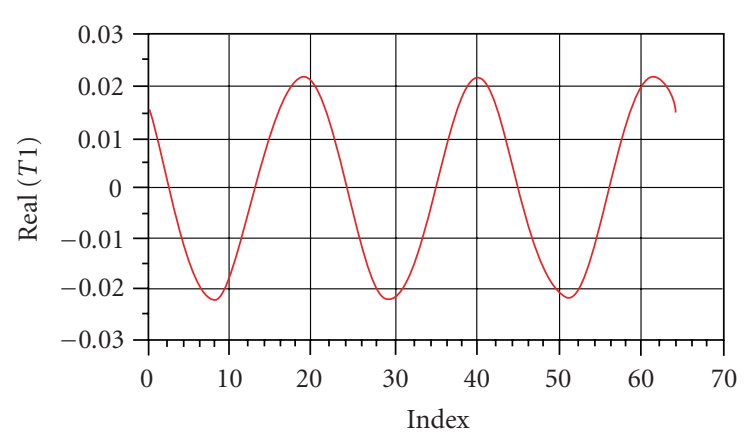

I-channel signal (after IFFT)

(b)

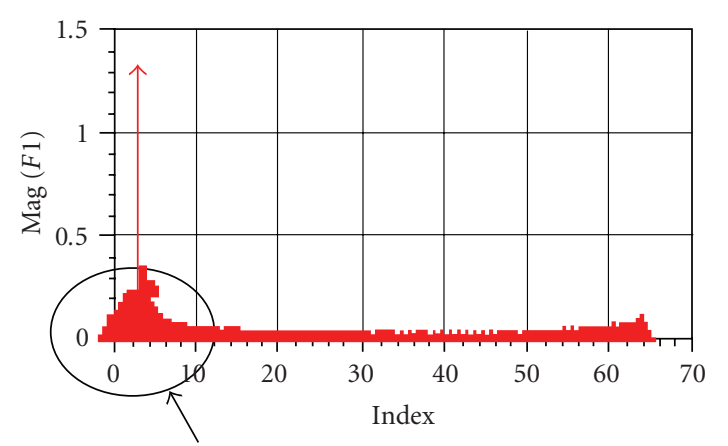

Recovered symbol contains ICI

(d)

Figure 2: ICI effects on OFDM signal.

In Figure 1, the configuration is optimal since no offset is present between the receiver and the transmitter. However, in Figure 2, an offset of $\delta_{f}$ is introduced and causes the different subcarriers to interfere.

The amount of shift might either be an integer multiple of the subcarrier spacing $\Delta_{f}$, in which case, the configuration would be similar to Figure 1. However, the received data symbols are now in the wrong position and so this will induce a bit-error rate of 0.5 [1]. However, the orthogonality among the subcarriers is still maintained. Indeed, the spectrum would now become

$$
S(f)=\sum_{k=0}^{N-1} S_{k} \sin c\left(\frac{f+C \Delta_{f}-\left(f_{0}+k \Delta_{f}\right)}{\Delta_{f}}\right),
$$

where $C$ is an integer constant.

The shift does not affect the location of the points of intersection of the different $\sin c$ waveforms that constitute the spectrum of the OFDM symbol. Orthogonality is thus preserved. If the shift is not an integer multiple of the carrier spacing, then we end up with interference among the different subcarriers. This interference may be quantified in the following way:

$$
I_{l}=\sum_{k=0, k \neq 1}^{N-1} S_{k} \sin c\left(\frac{\delta_{f}+(l-k) \Delta_{f}}{\Delta_{f}}\right) .
$$

It represents the amount of contribution other carriers have on the waveform associated with carrier $l$. So, $I_{l}$ is obtained by evaluating $S(f)$ at the frequency of interest, namely, the one associated with subcarrier $l$. Note that if the shift is zero or an integer multiple of the carrier-spacing, the aforementioned interference term would evaluate to zero. A nonzero value for $I_{l}$ can be interpreted as a loss of orthogonality of the basis functions because this would suggest that the projection of the waveform associated with carrier $l$ has nonzero components in the direction of the waveforms associated with the other carriers. In this sense, ICI is also referred to as the effect of loss of orthogonality. We will see this effect more in details in Section 5 in the context of phase noise.

\section{EFFECTS OF PHASE NOISE}

Signals are usually transmitted in the radio frequency range; but this is not compatible with the range of frequencies over which modulators/demodulators operate. Indeed, they operate at a much lower frequency usually referred to as the intermediate frequency. Converting the RF frequency to IF is thus crucial for proper demodulation of the signal. This down-conversion is performed by local oscillators which turn out to have the detrimental effect of introducing noise that will be superimposed on the received signal [4]. 
The introduced noise is caused by random variations of the phase about the steady-state sinusoidal waveform. In what follows, we assume that the transmitted OFDM signal has the same form as mentioned before, that is,

$$
s(t)=\sum_{k=0}^{N-1} S_{k} e^{j 2 \pi\left(f_{0}+k \Delta_{f}\right) t}
$$

In ideal conditions (if the local oscillator does not introduce noise), the received signal would be of the following form:

$$
r(t)=\sum_{k=0}^{N-1} H_{k} S_{k} e^{j 2 \pi\left(f_{0}+k \Delta_{f}\right) t}
$$

where $H_{k}$ denotes the complex frequency response of the channel in the $k$ th subchannel.

On the other hand, if the local oscillator is not ideal, we need to take into account the effect of noise. The latter is modeled as a complex random process and denoted by $e^{j \varphi(t)}$. We get the following received signal:

$$
\begin{aligned}
r(t) & =\sum_{k=0}^{N-1} H_{k} S_{k} e^{j 2 \pi\left(f_{0}+k \Delta f\right) t} \\
& =\sum_{k=0}^{N-1} R_{k} e^{j 2 \pi\left(f_{0}+k \Delta f\right) t} e^{j \phi(t)},
\end{aligned}
$$

where $R_{k}=H_{k} S_{k}$.

At the receiver, we project the received signal over each one of the possible carrier waveforms to determine the demodulated carrier amplitudes. This is translated in the following:

$$
\begin{aligned}
A_{l} & =\frac{1}{T_{s}} \int_{0}^{T_{s}} r(t) e^{-j 2 \pi\left(f_{0}+l \Delta f\right) t} d t \\
& =\frac{1}{T_{s}} \int_{0}^{T_{s}} e^{j \phi(t)} \sum_{k=0}^{N-1} R_{k} e^{j 2 \pi(k-l) \Delta_{f} t} d t
\end{aligned}
$$

where $T_{s}$ denotes an OFDM symbol time and is equal to $T_{s}=$ $1 / N \Delta_{f}$ and $N$ is equal to the number of different subcarriers. In a practical design, the afore-mentioned integral would be replaced by a summation [5], but the authors decided to use the integral because it makes the derivation closer to intuition even though nothing would change if they replace it by a summation.

The afore-mentioned expression denotes the projection of the received signal on the waveform associated with the carrier:

$$
2 \pi f_{1}=2 \pi f_{0}+2 \pi l \Delta_{f}
$$

The perturbations introduced by the random process $\varphi(t)$ are assumed to be relatively small so that the following approximation is justified:

$$
e^{j \phi(t)} \approx 1+j \phi(t)
$$

Now, we can write $A_{l}$ in the following way:

$$
\begin{aligned}
\frac{1}{T} \int_{0}^{T_{s}}(1+j \phi(t)) \sum_{k=0}^{N-1} R_{k} e^{j 2 \pi(k-l) \Delta_{f} t} d t \\
=\frac{1}{T_{s}} \sum_{k=0}^{N-1} R_{k} \int_{0}^{T_{s}} e^{j 2 \pi(k-l) \Delta_{f} t} d t \\
\quad+\frac{1}{T_{s}} \sum_{k=0}^{N-1} R_{k} \int_{0}^{T_{s}} j \phi(t) e^{j 2 \pi(k-l) \Delta_{f} t} d t .
\end{aligned}
$$

The second term will be denoted by $Y_{l}$.

This separation of the two terms is justified by the fact that the first part is what we would have received in the absence of noise while the second term includes the effect of noise.

Now, we write

$$
\frac{1}{T_{s}} \sum_{k=0}^{N-1} R_{k} \int_{0}^{T_{s}} j \varphi(t) e^{j 2 \pi(k-l) \Delta_{f} t} d t
$$

in the following way:

$$
\frac{1}{T_{s}} \sum_{k=0, k \neq l}^{N-1} R_{k} \int_{0}^{T_{s}} j \phi(t) e^{j 2 \pi(k-l) \Delta_{f} t} d t+\frac{R_{l}}{T_{s}} \int_{0}^{T_{s}} j \phi(t) d t .
$$

The second term of the afore-mentioned summation represents the contribution of the $l$ th carrier to the summation and is the one that we expect since we are projecting the received signal on the waveform associated with the $l$ th carrier. It evaluates to $j \phi_{0} R_{l}$, where $\varphi_{0}$ is a real constant that results from the integration.

We note that the constant $\varphi_{0}$ is independent of $l$ and so it is the same for all symbols of the signal constellation. We refer to this error as the CPE, which is directly proportional to the received amplitude $R_{l}$ of carrier $l$. We conclude that the physical meaning of this contribution is a rotation of the signal constellation by an angle equal to

$$
\phi_{0}=\frac{1}{T_{s}} \int_{0}^{T_{s}} j \phi(t) d t .
$$

We now characterize the other term that contributes to the previous summation, namely,

$$
\frac{1}{T_{s}} \sum_{k=0, k \neq l}^{N-1} R_{k} \int_{0}^{T_{s}} j \phi(t) e^{j 2 \pi(k-l) \Delta_{f} t} d t
$$

This term represents the remaining $N-1$ contributions of the other carriers. This contribution consists in first shifting the spectrum of $\varphi(t)$ by $22 \pi(k-l) \Delta_{f}$ and then scaling it by the received amplitude of the corresponding carrier. This amplitude is complex and thus this noise term will also be complex. It is commonly referred to as ICI or loss of orthogonality [6]. This is due to the fact that the orthogonal property of the basis functions that was used to produce the OFDM signal is no longer preserved. Indeed, the projection of the received signal on the waveform associated with the 
lth subcarrier now contains a new term which is completely random. Because of this randomness, it cannot be corrected.

More generally, we define $\varphi_{m}$ to be equal to

$$
\phi_{m}=\frac{1}{T_{s}} \int_{0}^{T_{s}} \phi(t) e^{j m \Delta_{f} t} d t .
$$

The noise effect can then be represented by the following matrix:

$$
\begin{aligned}
{\left[\begin{array}{c}
Y_{0} \\
Y_{1} \\
Y_{2} \\
\vdots \\
Y_{N-2} \\
Y_{N-1}
\end{array}\right]=j\left[\begin{array}{cccccc}
\varphi_{0} & \varphi_{1} & \varphi_{2} & \cdots & \varphi_{N-2} & \varphi_{N-1} \\
\varphi_{-1} & \varphi_{0} & \varphi_{1} & \cdots & \varphi_{N-3} & \varphi_{N-2} \\
\varphi_{-2} & \varphi_{-1} & \varphi_{0} & \cdots & \varphi_{N-4} & \varphi_{N-3} \\
\vdots & \vdots & \vdots & \vdots & \vdots & \vdots \\
\varphi_{-(N-2)} & \varphi_{-(N-3)} & \varphi_{-(N-4)} & \cdots & \varphi_{0} & \varphi_{1} \\
\varphi_{-(N-1)} & \varphi_{-(N-2)} & \varphi_{-(N-3)} & \cdots & \varphi_{-1} & \varphi_{0}
\end{array}\right] } \\
\times\left[\begin{array}{c}
R_{0} \\
R_{1} \\
R_{2} \\
\vdots \\
R_{N-2} \\
R_{N-1}
\end{array}\right] .
\end{aligned}
$$

The diagonal of the afore-mentioned matrix is constant because, as was explained before, this constitutes the CPE, which is the same for all carriers. The off-diagonal elements are the weighting coefficients that multiply each of the complex amplitudes of the received signals. The nomenclature intercarrier interference is thus clear from the structure of the afore-mentioned matrix.

$\varphi_{0}$ can be considered to be the output of a filter with the following impulse response:

$$
h(t)=\frac{\operatorname{rect}\left(t / T_{s}\right)}{T_{s}} .
$$

The impulse response is zero except for a rectangle of height $1 / T_{s}$ for a duration of $T_{s}$ seconds.

Taking the Fourier transform of $h(t)$, we get

$$
H(f)=\sin c\left(\frac{f}{\Delta_{f}}\right)
$$

It is very common to assume that the random process is a zero-mean Gaussian process. From this, we conclude that the random variables $\varphi_{m}$ are also Gaussian zero-mean. To fully characterize these random variables, we need to compute their variances.

For $\varphi_{0}$, we find that its power spectral density is given by $|\Phi(f) H(f)|^{2}$, where $\Phi(f)$ denotes the power spectral density of $\varphi(t)$. Its variance may then be computed as follows:

$$
\begin{aligned}
\sigma_{0}^{2} & =\int_{-\infty}^{\infty}|\Phi(f) H(f)|^{2} d f \\
& =\int_{-\infty}^{\infty} \sin c^{2}\left(\frac{f}{\Delta_{f}}\right)|\Phi(f)|^{2} d f .
\end{aligned}
$$

More generally, $\varphi_{m}$ is a zero-mean Gaussian random variable with variance equal to

$$
\sigma_{0}^{2}=\int_{-\infty}^{\infty} \sin c^{2}\left(\frac{f}{\Delta_{f}}\right)\left|\Phi\left(f-m \Delta_{f}\right)\right|^{2} d f .
$$

In fact, CPE can be accounted for as will be described in Section 7. The component we are left with is ICI.

We rewrite it for ease of reference:

$$
\frac{1}{T_{s}} \sum_{k=0, k \neq l}^{N-1} R_{k} \int_{0}^{T_{s}} j \phi(t) e^{j 2 \pi(k-l) \Delta_{f} t} d t .
$$

Using the afore-described filter, this can be rewritten as

$$
\frac{1}{T_{s}} \sum_{k=0, k \neq l}^{N-1} R_{k} \int_{-\infty}^{\infty} j \Phi\left(f-(k-l) \Delta_{f}\right) \sin c\left(\frac{f}{\Delta_{f}}\right) d f .
$$
get

We make the change of variable $u=f-(k-l) \Delta_{f}$ and

$$
\mathrm{ICI}=\frac{1}{T_{s}} \sum_{k=0, k \neq l}^{N-1} R_{k} \int_{-\infty}^{\infty} j \Phi(u) \sin c\left(\frac{u}{\Delta_{f}}+k-l\right) d u .
$$

We assume that the $k$ th subcarrier has average power $=2$. We can then compute the power of the ICI term and find it to be equal to

$$
\sum_{k=0, k \neq l}^{N-1} \sigma_{k}^{2} \int_{-\infty}^{\infty}|\Phi(u)|^{2} \sin c^{2}\left(\frac{u}{\Delta_{f}}+k-l\right) d u .
$$

From the afore-mentioned expression, we can see that if the power spectral density of $\varphi(t)$ is decreasing with frequency, then the major contribution to ICI will be that of subcarriers that are near the subcarrier of interest. We can also say that subcarriers near the center of the frequency band will suffer more from ICI than subcarriers at the edge of the band because contribution from adjacent subcarriers will be higher in the former case. In [5], it is stated that subcarriers near the center of the frequency band will be subject to more interference than subcarriers at the band edge by up to a factor of two.

Another important observation is that interference increases as the spacing between the subcarriers decreases.

From the power of the ICI, we can compute the noiseto-signal ratio. For a subcarrier near the middle of the band, assuming equal power on all subcarriers, the noise-to-signal ratio is given in [2] as

$$
\frac{\mathrm{ICI}^{2}}{\sigma_{l}^{2}}=2 \sum_{k=0}^{N / 2-1} \sigma_{k}^{2} \int_{-\infty}^{\infty}|\Phi(u)|^{2} \sin c^{2}\left(\frac{u}{\Delta_{f}}+k-l\right) d u
$$

We have seen that the effect of ICI increases as the relative spacing of the subcarriers decreases. So in this case, ICI effect may be considered small as compared to that of CPE. However, ICI is still present because the symbols in the constellation tend to have the shape of a cloud which depicts 
the random perturbation introduced by ICI. However, we can appreciate more the effect of CPE because it can be seen that the constellation has undergone a rotation by a constant angle. CPE can be corrected as will be briefly discussed in Section 7 and so after removing its effect.

\section{CORRECTION}

We have previously mentioned that CPE is the part of phase noise that can be accounted for and thus can be corrected.

The ability to do so stems from the fact that the effect of $\mathrm{CPE}$ is a simple rotation of the signal's constellation by a fixed angle. Thus, if we can estimate that angle, then correcting for CPE becomes a simple task as it would only consist in rotating back the constellation by the same angle.

One way to estimate the angle is by measuring the phase variation of a pilot subcarrier and subtracting that rotation from all subcarriers. The authors in [7] use an extended Kalman filter to present an algorithm for pilot-based CPE estimation.

Another way would consist in using differential phase modulation between the subcarriers. This method would, however, introduce a noise performance penalty when compared to coherent demodulation. The idea behind this technique is to let one subcarrier have a fixed phase and to let the information in the other subcarriers be encoded in their relative phase differences.

In [6], the authors propose a new algorithm for CPE correction.

Recall that

$$
\begin{aligned}
A_{t}= & \frac{1}{T_{s}} \sum_{k=0}^{N-1} R_{k} \int_{0}^{T_{s}} e^{j 2 \pi(k-l) \Delta_{f} t} d t \\
& +\frac{1}{T_{s}} \sum_{k=0, k \neq}^{N-1} R_{k} \int_{0}^{T_{s}} j \phi(t) e^{j 2 \pi(k-l) \Delta_{f} t} d t \\
& +\frac{R_{l}}{T_{s}} \int_{0}^{T_{s}} j \phi(t) d t,
\end{aligned}
$$

which can be written as

$$
A_{t}=\frac{1}{T_{s}} \sum_{k=0}^{N-1} R_{k} \int_{0}^{T_{s}} e^{j 2 \pi(k-l) \Delta_{f} t} d t+\mathrm{ICI}+\mathrm{CPE} .
$$

The first term on the left-hand side as well as ICI are both zero-mean. So, the authors of [6] suggest that we can get an estimate of the CPE for the lth OFDM symbol by finding the mean of the phase rotations associated with the subchannels of an OFDM symbol (because ICI and the first term have zero mean, they will not affect the expectation of $A_{l}$ for all $l$, and so we can have an estimate of the CPE by averaging over all the $A_{l}$ ). However, some subchannels may suffer from a low SNR, and thus including their effect in the CPE estimation will result in undesirable errors. These channels (for which SNR falls below a certain threshold) are discarded. We will denote by $N_{d}$ their number. The $A_{l}$ above the threshold will then be passed through a frequency equalizer to remove the effect of the channel. Note that this assumes that we already know the channel's characteristics. The equalized version of the $A_{l}$ is then passed through a slicer and we perform an averaging over the difference of the equalized version and the sliced version of the $A_{l}$.

This can be written as

$$
\mathrm{CPE}_{\text {estimate }}=\frac{1}{N_{d}} \sum_{l, \mathrm{H}_{l} \geq \text { threshold }}\left(A_{l, \text { equalized }}-A_{l, \text { sliced }}\right) .
$$

More generally, a tracking sequence occupying OFDM symbol $N_{t}\left(N_{t}\right.$ is generally set to 0$)$ is sent to allow a proper detection of the start of the OFDM frame and thus a proper removal of the cyclic prefix discussed previously. The above $\mathrm{CPE}$ estimation needs to be carried out for each symbol. CPE variations are usually very slow and the channel estimation procedure performed by the frequency equalizer remains the same unless a new tracking sequence is sent. If the block associated with the training sequence turns to have a high $\mathrm{CPE}$ estimate $\left(\mathrm{CPE}_{N_{t}}\right)$, then it will affect the channel phase estimation as described in [6]. This needs to be taken into account in the subsequent estimation of the CPE of the symbols. The authors in [6] use a moving average filter to remove the offset that might be introduced.

The algorithm proposed by the author can be formulated in the following way.

(1) Select the subchannels that meet the threshold criteria (SNR above a certain critical value).

(2) Calculate the mean of the previous CPE estimates that are in the moving average filter. We call this mean $\overline{\mathrm{CPE}_{m}}$.

(3) Use $\overline{\mathrm{CPE}_{m}}$ to update the phase of the coefficients $\left(C_{m-1, l}\right)$ of the frequency equalizer. This would prevent the occurrence of the offset previously discussed. The update can be described as follows:

$$
C_{m, l}=C_{m-1, l}+\overline{\mathrm{CPE}_{m}} \quad \text { for } 0 \leq l \leq N-1 .
$$

(4) Get the new estimate for

$$
\mathrm{CPE}_{\text {estimate }}=\frac{1}{N_{d}} \sum_{l, H_{l} \geq \text { threshold }}\left(A_{l, \text { equalized }}-A_{l, \text { sliced }}\right) .
$$

(5) Move the previous value in the moving average filter to calculate $\overline{\mathrm{CPE}_{m+1}}$.

\section{CONCLUSION}

OFDM is considered as a potential candidate for nextgeneration cellular systems. Despite all the advantages it provides in terms of ISI suppression and efficient bandwidth use, it suffers from the detrimental effects introduced by frequency offset and phase noise. The interference among the subcarriers causes the system to loose its orthogonality. We have seen that ICI places a limit on the carrier spacing because it increases as the spacing decreases. Local oscillators introduce phase noise that will be superimposed on the received signal. Many algorithms have been proposed to correct the CPE part of the phase noise. We have presented 
one algorithm based on averaging previous estimates of the CPE to get a better future estimate. The algorithm tracks well the variations of the CPE even though it usually varies slowly. On the other hand, ICI has a random structure and mitigating its effects remains a challenge.

\section{REFERENCES}

[1] M. Mourad, H. Salem, and B. Ridha, "Analysis of frequency offsets and phase noise effects on an OFDM 802.11 g transceiver," in Proceedings of the 4th International Workshop on Wearable and Implantable Body Sensor Networks (BSN '07), vol. 13, Aachen, Germany, March 2007.

[2] S. K. Bassam, "Frequency Synchronization Errors and Effects of Phase Noise on OFDM Systems," EE359-Wireless Communications Class Projects Stanford University, 2004.

[3] G. Pujolle, Les Réseaux, Eyrolles, Paris, France, 4th edition, 2000.

[4] H. Salem, "Contribution à la modélisation des câbles monotones par éléments finis," thesis report ENIT, January 2000.

[5] A. R. S. Bahai and B. R. Saltzberg, Multi-Carrier Digital Communications. Theory and Applications of OFDM, Kluwer Academic Publishers/Plenum, New York, NY, USA, 1999.

[6] V. S. Abhayawardhana and I. J. Wassell, "Common phase error correction for OFDM in wireless communication," in Proceedings of the 3rd International Symposium on Communication Systems, Networks and Digital Signal Processing (CSNDSP '02), Staffordshire, UK, July 2002.

[7] D. Petrovic, W. Rave, and G. Fettweis, "Phase noise suppression in OFDM using a Kalman filter," in Proceedings of the 6th International Symposium on Wireless Personal Multimedia Communications (WPMC '03), vol. 3, pp. 375-379, Yokosuka, Japan, October 2003. 

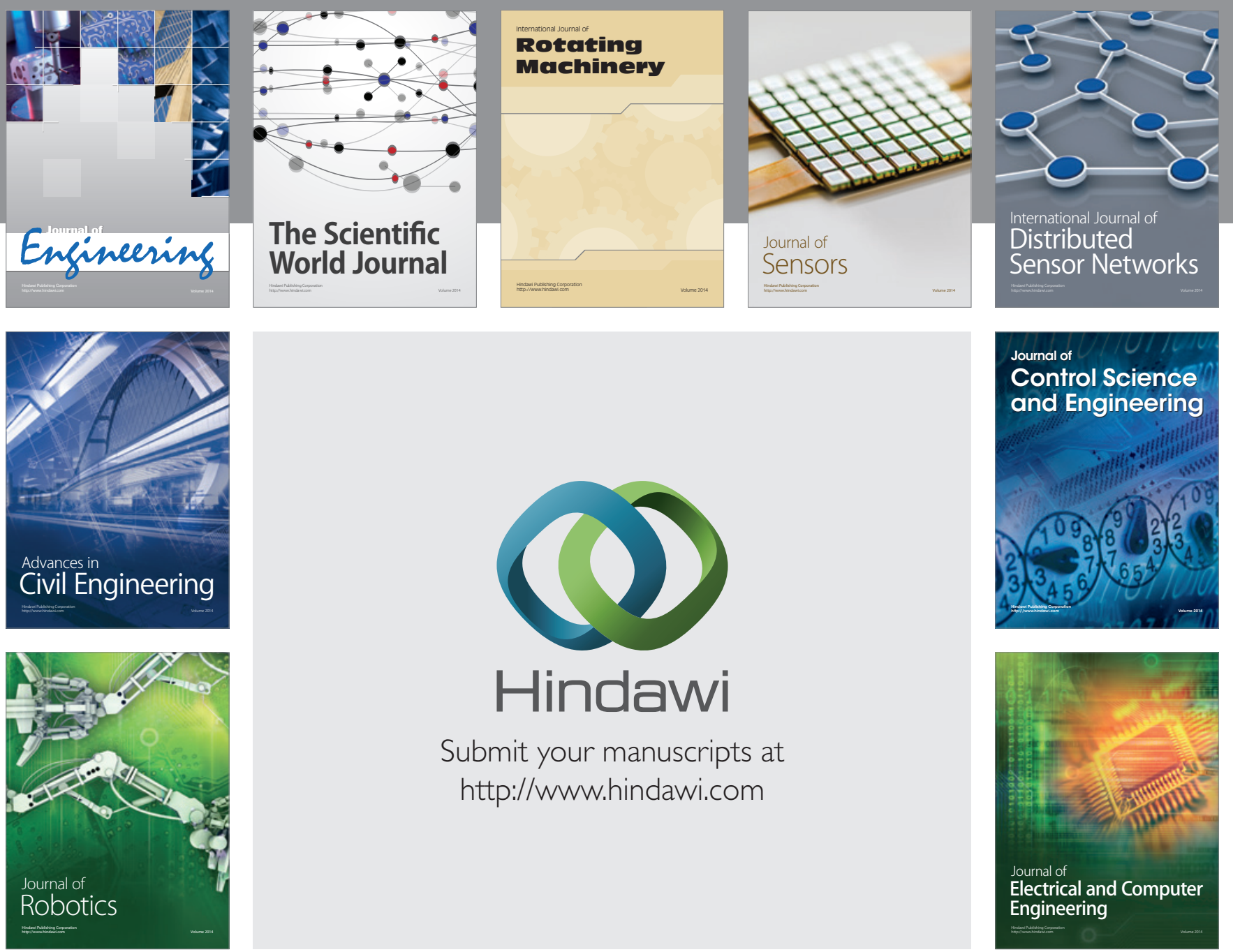

Submit your manuscripts at

http://www.hindawi.com
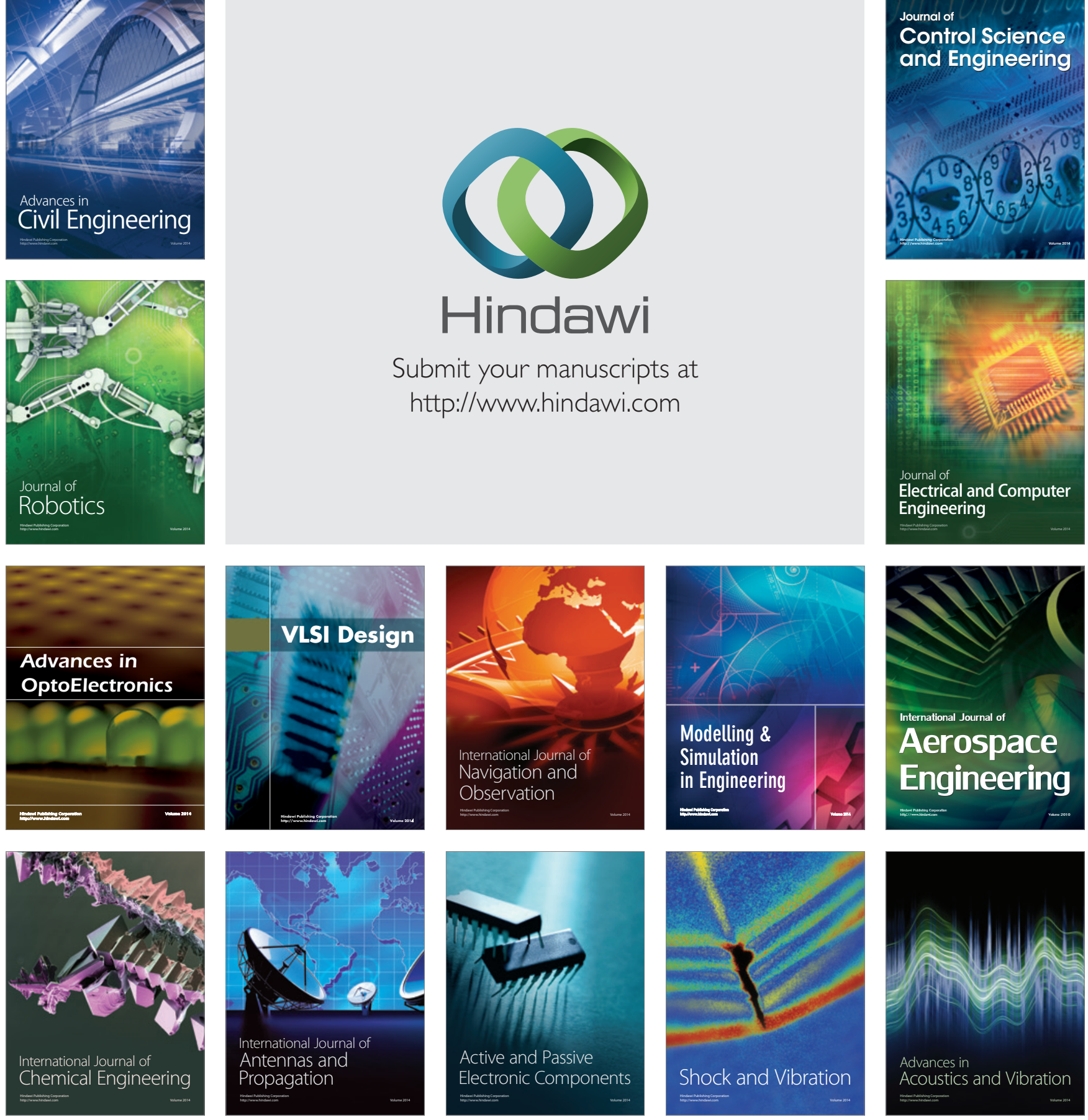